\title{
L'expression de personne en Anambé du Cairari
}

Maria Risolêta Silva Julião ${ }^{1}$

\section{Resumée}

Cet article verse sur le fonctionnement du système de marques de personne en Anambé du Cairari en prenant en compte la valence du verbe, le type de participant (A, O ou S) et le mode verbal. Il présente aussi des particularités de la forme pronominale de la troisième personne anambé.

Mots-clés: Morphosyntaxe, marques de personne, valence verbale.

\begin{abstract}
This paper is about the personal system of Anambé do Cairari. The analysis is based primarily on the verbal valence, the type of participants (A, O or $\mathrm{S}$ ) and the verbal mode. The paper also focus on the particular characteristics of the third person pronoun in Anambé.
\end{abstract}

Keywords: Morpho-syntax, personal markers, verbal valence.

\section{Considérations initiales ${ }^{2}$}

Dans cet article nous nous concentrons sur les marques de personne en Anambé du Cairari ${ }^{3}$, une langue appartenant à la sous-branche $\mathrm{V}$ de la famille Tupí-Guaraní (Rodrigues et Cabral 2002; Julião 2005), qui a été parlé comme langue maternelle jusqu'à la moitié du dernier siècle par le groupe également connu sous le nom d'Anambét. Compte tenant de l'appartenance de l'Anambé du Cairari à la sous-branche V, à côté de l'Araweté et de l'Asuriní du Xingu, on prendra des données de ces langues pour les contrastés, en soulignant les spécificités du système personnel de l'Anambé.

Les données qui supportent l'analyse ont été obtenues auprès de deux dernières locutrices de l'Anambé, Durica et Tapira, mère et fille, entre 1990

\footnotetext{
${ }^{1}$ Universidade Federal do Pará ; Laboratório de Línguas Indígenas-UnB.

${ }^{2}$ Je remercie Aryon D. Rodrigues et Ana Suelly Arruda Câmara Cabral pour leurs observations et pour l'incitation à la publication de cet article.

3 Cabral e Rodrigues (2002) appellent la langue registrée au bord du Cairari comme Anambé do Cairari pour la différencier de celle registrée par Ehrenreich en 1894, dans la région du Tocantins. Nous adoptons ici la dénomination suggérée pour les premiers.

${ }^{4} \mathrm{La}$ graphie pour les noms des peuples indigènes et pour les langues qu'ils parlent suivi la convention proposée par l'Associação Brasileira de Antropologia.
} 
et 2004. Tapira vit encore dans la Terre Indigène Anambé, situé sur la rive droite de la rivière Cairari, Municipalité de Moju, sud-est du Pará. Durica est morte en 2004.

L'article s'organise autour des moyens parmi lesquels la catégorie de personne s'exprime en Anambé du Cairari aussi bien que de la distribution des différents ensembles des marques de personne dans les structures de la langue. D'abord nous ferons quelques considérations générales sur les marques de personne; en suite nous présenterons l'ensemble des pronoms, suivi de celui des préfixes et, finalement, nous ferons des considérations sur le comportement de la troisième personne.

\section{Les marques de personne : considérations générales}

Il y a en Anambé du Cairari cinq ensembles de marques personnels parmi lesquels trois des pronoms et deux de préfixes, dont la distribution est définie selon la valence du verbe, le type de participant $\left(\mathrm{A}, \mathrm{O}\right.$ ou $\left.\mathrm{S}^{5}\right)$ et le mode verbal.

Les données ont permis d'établir les paradigmes que l'on observe dans le tableau suivant:

Tableau 1 - Les marques de personne

\begin{tabular}{|c|c|c|c|c|c|c|}
\hline \multirow{2}{*}{} & \multicolumn{3}{|c|}{ Pronoms } & \multicolumn{3}{c|}{ Préfixes } \\
\cline { 2 - 7 } & Indépendants & \multicolumn{2}{|c|}{ Clitiques } & \multicolumn{2}{c|}{} \\
\cline { 2 - 7 } & & CL1 & CL2 & PRF1 & PRF2 & PRF3 \\
\hline $1 \mathrm{~s}$ & ja & iha & iha & a- & te- & \\
\hline 1 exc & ra & ura & uru & uru- & uru- & \\
\hline 1 inc & jana & jene & jana & xa- & tire- & \\
\hline $2 \mathrm{~s}$ & na & ne & ere & ere- & ere- & e- \\
\hline $2 \mathrm{pl}$ & pẽ & pe & pe & pe- & pe- & pe- \\
\hline 3 & wỹ & & u & & $\emptyset-$ & \\
\hline
\end{tabular}

L'Anambé n'a qu'une seule forme pour la troisième personne, soit en fonction de sujet soit d'objet, singulier ou pluriel. Il s'agit du morphème $\tilde{u}$, qui est, en fait, une sorte de déictique. Ces particularités distinguent l'Anambé

\footnotetext{
${ }^{5}$ On utilisera les notations de Dixon (1994) référents aux rôles sémantico-syntaxiques associés aux participants qui expriment respectivement les fonctions de sujet des phrases transitives (A), de sujet des phrases intransitives $(\mathrm{S})$ et d'objet des phrases transitives $(\mathrm{O})$.
} 
du Cairari de l'Araweté et de l'Asuriní du Xingu qui présentent des formes préfixales de troisième personne, ainsi que de déictiques. D'après Monserrat et al. (1998:6), l'Asuriní en plus distingue une forme féminine $\tilde{e}$, une forme singulière masculine $g a$ et une forme plurielle commune $g y^{6}$.

La langue présente une autre forme pour marquer une troisième personne, mais celles-ci a une fonction emphatique, excepté quand il marque l'un des arguments constituant une équation $(\mathrm{X}=\mathrm{Y})$, où la troisième personne est toujours Y.

La première personne du pluriel présente deux formes, l'une inclusive et l'autre exclusive, aussi pour les pronoms que pour les PRF1 et pour les PRF2. La première inclusive est employée quand le locuteur se réfère à luimême et à l'interlocuteur; l'exclusive, quand le locuteur se réfère à lui-même et à d'autres individus, en excluant l'interlocuteur. Les PRF3 présentent une seule catégorie de personne, la deuxième.

\section{Les pronoms}

L'ensemble des pronoms comprend des pronoms indépendants et des pronoms clitiques. Les indépendants se trouvent a) dans des constructions équatives (exemples (1) et (2)), b) dans les prédicats locatifs (exemple (3)), c) comme sujet emphatique dans les constructions indépendantes à l'Indicatif I avec des verbes transitifs (exemple (4) et avec un des sous-ensembles des verbes intransitifs dont la référence personnelle est redondante, devant ainsi être doublement marqué (exemple (5)). Avec des verbes transitifs, ils sont optionnels, mais obligatoires avec les verbes intransitifs.

kujỹ rixa jana
femme $\mathrm{Neg}^{7} \quad$ inc
'Nous ne sommes pas (des) femmes.'

\footnotetext{
${ }^{6}$ Cf. aussi Pereira (2009:131).

${ }^{7}$ Abréviations: CLI=Clitic personnel de l'ensemble 1; CL2 = Clitic personnel de de l'ensemble 2; Cor=Coréférentiel; Dat=Datif; Dem=Demonstratif; Dir=Direction Foc=Focus; Ger=Gerondif; Imp=Impératif; Inf=Inférentiel; Neg=Négation; PFR1=Préfixe personnel de l'ensemble 1; PRF2= Préfixe personnel de l'ensemble 2; PRF3 = Préfixe personnel de l'ensemble 3; R1=Préfixe que marque la contigüité du déterminant d'un thème dépendant; Rel=Relationel; Subj=Subjonctif; Trs=Translatif; $1 \mathrm{~s}=$ Première personne du singulier; 1inc=Première personne pluriel inclusive; 1exc=Première personne exclusive; $2 \mathrm{~s}=$ Deuxième personne singulier; $2 \mathrm{pl}=$ Deuxième personne pluriel; $3=$ Troisième personne; $1=$ Première personne; $2=$ Deuxième personne; $13=$ Première personne du pluriel exclusive.
} 
(2)

$\begin{array}{llll}\text { Aipã } & \text { iha } & \emptyset \text {-tuty } & \boldsymbol{w} \tilde{\boldsymbol{y}} \\ \text { Aipã } 1 \mathrm{~s} & \text { Rel-oncle } & 3 \\ \text { 'Aipã était mon oncle.' }\end{array}$

(3)

$$
\begin{aligned}
& \text { na } \quad \text { ru-mẽ Najã } \\
& 2 \mathrm{~s} \quad \text { Dem-dans ce moment Najã } \\
& \text { 'Tu es là dans ce moment, Najã?' } \\
& \text { 'Tu es là, Najã?' }
\end{aligned}
$$

(4) manio (ja) a-kyty

manioc 1s 1s-râper

'Moi, je râpe le manioc'

'Je râpe le manioc.'

(5)

$$
\begin{aligned}
& \text { a-pukã ja } \\
& \text { 1s-rire 1s } \\
& \text { 'Je ris, moi.' } \\
& \text { 'Je ris.' }
\end{aligned}
$$

Les pronoms clitiques se manifestent comme l'on voit en suite. Les CL1 se manifestent toujours de la même façon que le nom: immédiatement placés devant une racine nominale, postpositionnelle ou verbale préfixée par le relationnel $\{\mathrm{r}-\}: \mathrm{r}-, \emptyset$ - . Avec les deux premières types de racine ils référent: a) le déterminant des noms (exemples (6) et (7)), b) l'objet des postpositions (exemples (8) et (9)).

(6)

$$
\begin{aligned}
& \text { iha r-apy } \\
& 1 \mathrm{~s} \quad \text { REL-maison } \\
& \text { 'Il y a une maison à moi' } \\
& \text { 'Ma maison.' }
\end{aligned}
$$

$$
\begin{aligned}
& \text { ne } \quad \text {-hy } \\
& \text { 1s REL -mère } \\
& \text { 'Il y a ta mère.' } \\
& \text { 'Ta mère.' }
\end{aligned}
$$


$(8)$

pyriru e-ru iha r-upa

sandale 2sImp apporter $1 \mathrm{~s}$ Rel-Dat

'Apporte une sandale pour moi.'

'Apporte-moi une sandale.'

(9)

$\begin{array}{lllll}\text { kurumĩ } & \tilde{u} & \text { ka } & \text { pe } & \emptyset \text {-puha } \\ \text { enfant } & 3 & \text { dormir } & 2 \mathrm{pl} & \text { Rel-Com } \\ \text { 'L'enfant dort avec vous.' } & \end{array}$

Avec les racines verbales les CL1 marquent: a) le sujet des verbes intransitifs à l' Indicatif II (exemple (10)) et au Subjonctif (exemple (11)), b) l'objet des verbes transitifs dans l'Indicatif I (exemples (12) et (13)) et dans le Gérondif (exemple (14)).

$\begin{array}{lll}\text { Catarina } & \text { r-enã } \quad \text { jene ho } \\ \text { Catarina } & \text { Rel-maison } & \text { linc aller } \\ \text { 'Nous sommes allés chez Catarina.' }\end{array}$

(11) kwa-ty ne eri mẽ iha ho me?ẽ Arara-raha Dem-Dir 2s venir Subj 1s aller regarder A-Dat 'Quand tu es venu dans cette direction, je suis allée regarder sur Arara.'

(12) ne Ø-hy jene r-arũ

2s Rel-mère linc Rel-attendre

'Ta mère nous attend.'

(13) jawãti uru Ø-u?u

chien lexc Rel-mordre

'Le chien nous a mordu.'

$\begin{array}{lllllll}\text { (14) } & \text { iha } & \text { ne } & \emptyset \text {-ihũ } & \text { ne } & \text {-jukã } & \text { na } \\ & 1 & 2 & \text { Rel-percer } & 2 & \text { Rel-tuer } & \text { ger }\end{array}$

'J'ai te percer pour te tuer.'

En plus, les CL1 référent le seul constituant des phrases elliptiques, telles que des réponses aux questions «Qui a fait cette robe ?», (exemple (10)), «Qui a pêché (ces poissons)?» (exemple (11)): 
(15)

$$
\begin{aligned}
& \text { iha } \\
& 1 \mathrm{~s} \\
& \text { 'Moi.' }
\end{aligned}
$$

(16) ura

$1 \mathrm{exc}$

'Nous.'

Les CL2 se combinent avec des verbes transitifs pour renvoyer à $\mathrm{A}$ dans les constructions indépendantes lorsque $\mathrm{O}$ est un élément pronominal CL1 :

$$
\begin{array}{llll}
\text { uru } & \text { ne } & \text { r-ekã } & \text { xa } \\
1 \operatorname{exc}_{\mathrm{A}} & 2 \mathrm{~s}_{\mathrm{O}} & \text { Rel-chercher } & \mathrm{Neg} \\
\text { 'Nous ne t'avons pas cherché.' }
\end{array}
$$

Reprenons ici le fonctionnement des CL1 en tant qu'objet dans les phrases à l'Indicatif I le contrastant avec l'Araweté et l'Asuriní du Xingu, compte tenant des relations que s'établissent entre les participants du discours quand de première et de deuxième personne.

Comme l'on observe, l'Anambé (exemples (18), (20), (22), (24)) se rapproche de l'Araweté (exemples (19), (21), (23), (25)) quant à l'usage des formes pour marquer $\mathrm{O}$, étant donné que les formes de l'Araweté correspondent au CL1 de l'Anambé 8 . Remarquons que pour marquer A, l'Araweté fait usage de la même série qui sert à marquer $\mathrm{O}$, tandis que l'Anambé utilise les formes CL2, conformément à se qu'on a vu ci-dessus.

$$
\begin{aligned}
& \text { iha ne } \emptyset \text {-ajỹ he } k u \text { ne } \emptyset \text {-nu } \\
& 1 \mathrm{~s}_{\mathrm{A}} \quad 2 \mathrm{~s}_{\mathrm{O}} \text { Rel-pousser } \quad 1 \quad \text { Foc } 2 \text { R1-frapper } \\
& \text { 'Je te pousse.' 'je t'ai frappé.' }
\end{aligned}
$$

$$
\begin{aligned}
& \text { uru ne } \emptyset \text {-ajy } \quad \text { ure } k u \text { ne } \emptyset \text {-piti } \\
& 1 \operatorname{exc}_{\mathrm{A}} 2 \mathrm{~s}_{\mathrm{O}} \text { Rel-pousser } 13 \quad \text { Foc } 2 \quad \text { R1-pincer } \\
& \text { 'Nous te poussons.' 'nous t'avons pincé.' }
\end{aligned}
$$

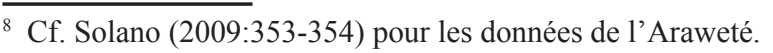


(22)

\begin{tabular}{llllll} 
ere $\quad$ iha & $r$-enu & $n e$ & $k u$ & he & $\emptyset$-piri \\
$2 \mathrm{~s}_{\mathrm{A}} \quad 1 \mathrm{~s}_{\mathrm{O}} \quad$ Rel-écouter & 2 & Foc & 1 & $\mathrm{R}^{1}$-pincer \\
'Tu m'écoutes.' & \multicolumn{3}{c}{ 'tu m'as pincé.' }
\end{tabular}

(24)

$\begin{array}{llll}\text { pe } & \text { ura } & \text { r-enu } & \text { ure } \text { r-et } f a \text { ku } p \tilde{z} \\ 2 \mathrm{pl}_{\mathrm{A}} & 1 \text { exc }_{\mathrm{O}} & \text { Rel-écouter } & 13 \mathrm{R}^{1} \text {-voir Foc } 23 \\ \text { 'Vous nous écoutez.' } & \text { 'vous nous avez vu.' }\end{array}$

De l'autre côté, si on les compare à l'Asuriní du Xingu, par exemple, on verra que différemment de l'Anambé cette langue dispose de préfixes spécifiques pour marquer l'objet, quand il s'agit de la relation entre une première personne $\mathrm{A}$ et une deuxième personne $\mathrm{O}$. Dan ce cas l'Assurini utilise les préfixes uru- e puru pour marquer respectivement une deuxième personne du singulier $\mathrm{O}$ (exemples (26)-(27)) et une deuxième personne plurielle O (exemples (28)-(29)) (Monserrat et al. 1998:11) ${ }^{9}$.

(je) uru-esak

'je t'ai vu.'

(je) a-puru-esak

'je vous ai vus.'
(27) (ure) uru-esak

'nous t'avons vu'

(29) (ure) uru-puru-esak

'nous vous avons vus.'

Pour ce que se réfère à la relation entre une deuxième personne $\mathrm{A}$ et une première personne O, l'Asuriní, selon Monserrat et al. (1998:11) ${ }^{10}$, différemment de l'Anambé du Cairari et de l'Araweté (cf. aussi Cabral e Solano 2006:55), utilise les formes pronominales ape e pejepe, placés après le prédicat pour marquer l'agent:

$$
\begin{aligned}
& \text { je r-esak ape } \\
& \text { 'você me viu' } \\
& \text { je r-esak pejepe } \\
& \text { 'vocês me viram' }
\end{aligned}
$$

ure r-esak ape

'você nos viu'

ure $r$-esak pejepe

'vocês nos viram'

\footnotetext{
$\overline{9}$ Cf. Cabral (2001) pour l'hypothèse sur le développement de ces morphèmes dans le cadre de la famille Tupí-Guaraní.

${ }^{10}$ Cf. aussi Pereira (2009:245).
} 


\section{Les préfixes personnels}

Pour les préfixes personnels, on a la distribution suivante. Les PRF1 se combinent uniquement avec des verbes dont ils marquent le sujet $\mathrm{S}$ ou $\mathrm{A}$. Dans le dernier cas, lorsque O est exprimé par un nominal (exemple (35)):

$$
\begin{aligned}
& \text { ere-je?e pipi?i } \\
& \text { 2s-parler peu } \\
& \text { 'Tu parles peu.' }
\end{aligned}
$$

$$
\begin{array}{ll}
\text { timoapỹ } & \boldsymbol{r e} \text {-mujã } \\
\text { panier } & \text { 2s-faire }
\end{array}
$$

'Tu fais un panier.'

Les PRF2 constitue l'ensemble des préfixes coréférentiels qui, en Anambé, a de formes pour toutes les personnes. En suivant Jensen (1998b:23), l'Anambé appartient, alors, à la catégorie des langues TupíGuaraní qui présentent un paradigme maximal de préfixes coréférentiels. Cette caractéristique est aussi présente en Araweté et en Asuriní du Xingu.

La forme de deuxième personne singulière ne correspondent pas à celle reconstruite par Jensen (1998a) pour le proto-Tupí-Guaraní. En considérant le fait que toutes les langues conservatrices de la famille ont une deuxième personne coréférentielle $e$-, il est fortement probable que l'Anambé du Cairari ait remplacé une ancienne forme $e$ - par la forme correspondent du PRF1. Quant à la forme pour la première personne du singulier et pour la première personne inclusive, leurs correspondances avec l'Asuriní du Xingu et l'Araweté sont remarquables.

En ce qui concerne la troisième personne coréférentielle, l'Anambé différemment de l'Araweté et de l'Asuriní du Xingu n'a pas une forme phonologiquement ouverte.

En Anambé les préfixes coréférentiels marquent: a) $\mathrm{S}$ dans les phrases intransitives au Gérondif si on a dans la phrase principale un verbe directionnel, comme aller ou venir (exemples (36)-(37)), b) le déterminant du nom coréférent au sujet dans les phrases indépendantes (exemples (38) et (39)) et c) le sujet des verbes auxiliaires (exemple (40)). Ils ne se combinent pas avec des verbes au mode Subjonctif, ni avec des postpositions. 
(36) xa-ho tire-purahã

linc-aller 1inc-danser

'Nous allons (pour) danser.'

a-jo ja te-je?ẽ

1s-venir 1s 1sCor-bavarder

'Je suis venue (pour) bavarder.'

(38) migau a-muрupu te-memy r-ира

bouillie 1s-faire-cuire 1s-fils Rel-Dat

'Je prépare de la bouillie pour mon fils (de femme).'

(39) maجarahypuhỹ ũ kyty Ø-py $\quad r$-aha

médicament 3 mettre 3Cor-pied Rel-Dat

'Elle a appliqué le médicament sur son propre pied.'

(40) manißo uru-kyty uru-enã

manioc 1 inc-râper linc-être.sans mouvement

'Nous sommes en train de râper le manioc.'

Les données suivantes présentent les PRF3 qui sont employés exclusivement avec le mode Impératif:

(41) e-karu

2sImp-manger

'Mange!'

(42) pe-karu

2plImp-manger

'Mangez!'

(43) $\quad$-jo $\quad$ xa

2sImp-pleurer Neg

'Ne pleure pas!' 
(44)

$$
\begin{aligned}
& \text { e-kwã } \quad \text { xa } \text { e-putz rumũ } \\
& \text { 2sImp-s'en aller Neg 2sImp-rester ici } \\
& \text { 'Ne t'en va pas, reste ici!' }
\end{aligned}
$$

\section{La troisième personne en Anambé}

La manifestation de la troisième personne est conditionnée par le type de verbe ou par le type de phrase - à prédicat verbal ou non-verbal.

Dans les phrases à prédicat verbal au Gérondif on utilise, aussi, les allomorphes du relationnel $\{\mathrm{i}-\}$ : i-, $\emptyset$-, pour marquer $\mathrm{O}$, si la racine verbale est transitive et appartient à la Classe II:

$$
\begin{array}{lll}
\tilde{u} & \emptyset \text {-ihũ } \quad \text { i-jukã na } \\
3 & \text { percer } & \text { Rel -tuer Ger } \\
\text { 'On l'a percé pour le tuer.' }
\end{array}
$$

$$
\begin{array}{lllll}
\text { pirã } & \tilde{u} & \text { pyhy } & \boldsymbol{i} \text {-?o } & \text { na } \\
\text { poisson } & 3 & \text { pêcher } & \text { Rel-nettoyer } & \text { ger }
\end{array}
$$

$$
\begin{aligned}
& \text { i-mihi na } \quad \text { i-?u na } \\
& \text { rel-griller ger rel-manger ger } \\
& \text { 'Il a pêché le poisson, (il) l'a nettoyé, (il) l'a grillé et (il) l'a mangé.' }
\end{aligned}
$$

Par contre, si la racine verbale appartient à la Classe I, la troisième personne y est exprimée par le morphème personnel $\tilde{u}_{\text {' }} 3$ ' :

$$
\begin{array}{lllll}
\text { a-ho } & \text { ja } & \tilde{\boldsymbol{u}} & \text { r-ekã } & \text { na } \\
\text { 1s-aller } & \text { 1s } & 3 & \text { Rel-chercher } & \text { Ger } \\
\text { 'Je vais (pour) le chercher.' }
\end{array}
$$

A l'Indicatif (exemple (51)-(53)) et au Subjonctif (exemple (54)) on utilise aussi le morphème $\tilde{u}$ pour marquer A. Dans le premier cas, comme l'on a déjà vu, $\tilde{u}$ se combine avec le pronom wỹ que fonctionne comme sujet emphatique. Optionnel avec des verbes transitifs, mais obligatoire avec un des sous-ensemble des verbes intransitifs dont la référence personnelle est redondant: 
(48)

$$
\begin{aligned}
& \text { (wỹ) } \tilde{\boldsymbol{u}} \text { iha } \emptyset \text {-ajỹ } \\
& 33 \text { 1s Rel-pousser }
\end{aligned}
$$

(49)

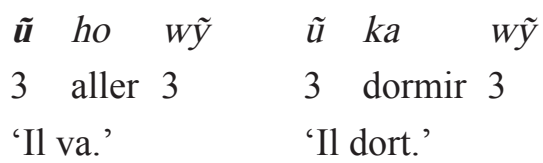

(51)
$\tilde{\boldsymbol{u}}$ emu?a mẽ
ma२arahypuhỹme?ẽhã-mũ
$w \tilde{y}$
3 étudier Subj
infirmier-Trs
3

'S'il étudie, il sera infirmier.'

Un fait intéressant c'est que le morphème $\tilde{u}$ reste obligatoirement présent, même lorsque $\mathrm{A}$ et $\mathrm{O}$ sont spécifiés par un nominal plein ${ }^{11}$ :

$$
\begin{aligned}
& \text { Maria } \tilde{\boldsymbol{u}} \text { pukapukã } \quad \text { w̃ } \\
& \text { Maria } 3 \text { rire aux éclats } 3 \\
& \text { 'Maria rit aux éclats.' }
\end{aligned}
$$

$$
\begin{aligned}
& \text { kuma?a mo } \quad \tilde{\boldsymbol{u}} \text { jukã } \\
& \text { Homme serpent } 3 \text { tuer } \\
& \text { 'L'homme a tué le serpent.' }
\end{aligned}
$$

$$
\begin{array}{lllll}
\text { Ronca jawã } & \tilde{\boldsymbol{u}} & \text { piro } & \text { ra?a } \\
\text { Ronca } & \text { jaguar } 3 & \text { écorcher } & \text { Inf } \\
\text { 'Ronca a écorché le jaguar.' }
\end{array}
$$

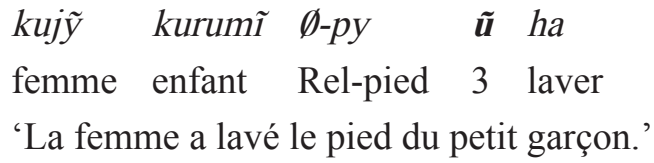

\footnotetext{
11 Les constructions avec des verbes intransitifs rappellent le cas du palau cité par Lemaréchal (1997:28-29) et l'Anambé pourrait s'inscrire parmi les langues où "il n'y a pas de relation directe possible entre un sujet constitué par un syntagme et un prédicat, mais une relation médiatisée".
} 


$\begin{array}{llllll}\text { Bacuri Juruti } & \emptyset-p o & \tilde{\boldsymbol{u}} & \text { kixi } & \text { mukũ } \\ \text { Bacuri Juruti } & \text { Rel-main } & 3 & \text { entailler } & \text { deux.fois } \\ \text { 'Bacuri a entaillé la main de Juriti deux fois.' }\end{array}$

Néanmoins, la présence d'un nominal plein dans les phrases transitives ainsi que dans les phrases intransitives, n'est pas obligatoire pour aboutir à une phrase bien formée. Le plus fréquent est l'absence du nominal. Ainsi, à la place de (52) nous aurons (60) :

$$
\begin{aligned}
& \tilde{u} \text { pukapukã } \\
& 3 \text { rire aux éclats } \\
& \text { 'Elle rit aux éclats.' }
\end{aligned}
$$

et à la place de (53) nous aurons (61) :

$$
\begin{aligned}
& \text { mo } \quad \tilde{u} \quad \text { jukã } \\
& \text { serpent } 3 \text { tuer } \\
& \text { 'Il a tué le serpent.' }
\end{aligned}
$$

Des constructions comme celles en (52)-(56) sont fréquemment le résultat d'une négociation entre la chercheuse et les consultantes où l'on essaye de contextualiser l'événement et d'identifier l'ordre des arguments. D'autres études sur la famille Tupí-Guaraní assignent l'usage facultatif des groupes nominaux (cf. Vieira 1993; Seky 2000; Leite 2001).

L'absence du nominal (kuma?a 'homme' en (58), par exemple) implique une certaine ambiguïté et d'autres interprétations sont possibles: "le serpent (il) tue" ou "le serpent l'a tué". Soulignons, néanmoins, que la consultante Tapira n'a pas accepté ces interprétations, en expliquant: "[le] serpent ne tue pas; il mord et les gens meurent". Ainsi, les informations contextuelles, biologiques, culturelles ou pragmatiques, dans ces cas, peuvent résoudre l'ambiguïté.

Quand il s'agit des verbes transitifs, un problème se pose: savoir quel est l'argument qui se marque auprès du verbe, celui du sujet ou celui de l'objet? Cependant, au vu des exemples suivants où la marque personnelle attachée au verbe est celle qui marque le sujet, on peut dire que dans les exemples 
(55)-(59) le constituant est celui du sujet, étant donné que le morphème $\tilde{u}$, dans ce contexte-ci, se substitue aux préfixes subjectifs

$$
\begin{aligned}
& \text { jawã a-piro ra?a } \\
& \text { jaguar 1s-écorcher Inf } \\
& \text { 'J'ai écorché le jaguar.' }
\end{aligned}
$$

$$
\begin{array}{llll}
\text { kurumĩ } & \emptyset-p y & \text { a-ha } & \text { raPa } \\
\text { enfant } & \text { Rel-pied } & \text { 1s-laver } & \text { Inf } \\
\text { 'J'ai lavé le pied du petit garçon.' }
\end{array}
$$

$$
\begin{aligned}
& \text { Juruti } \quad \text {-po } \quad \text { a-kixi raPa mukũ } \\
& \text { Juruti Rel-main 1s-entailler } \quad \text { Inf deux.fois } \\
& \text { 'J'ai entaillé la main de Juriti deux fois.' }
\end{aligned}
$$

Avec des phrases à prédicat non-verbal le theme ne reçoive pas de marque de personne. Il se combine avec le préfixe connu d'après Rodrigues ([1981] 2010)) comme préfixe relationnel $\{$ i- $\}$ : i-, $\emptyset$ - qui marque la noncontigüité syntaxique du déterminant, comme l'on voit dans les exemples ci-dessous:

$$
\begin{aligned}
& \text { i-aru jawatĩ } \\
& \text { Rel-méchant chien } \\
& \text { 'Il est méchant, le chien,' }
\end{aligned}
$$

(63) Ø-jahu Merã r-ekahĩ

Rel-neuf Merã Rel-hamac

'Il est neuf, le hamac de Merã.'

(64) Ø-tuwihauhu kuma?a Ø-namy

Rel-grand homme oreille

'Elle est grande, l'oreille de (cet) homme.' 


\section{Considérations finales}

Les données ici présentées révèlent que le système de marquage de personne de l'Anambé est assez riche, en distinguant les fonctions des arguments $\mathrm{A}, \mathrm{S}$ et $\mathrm{O}$ d'après le mode verbal. La langue présente un paradigme de préfixes coréférentiels qui contemplent toutes les personnes et se combine avec des verbes aussi bien qu'avec des noms. Nous avons aussi démontré que l'Anambé présente une seule forme pour exprimer une troisième personne, indépendamment du mode verbale.

L'ensemble de traits qui caractérise le système personnel de l'Anambé fait de lui une langue unique en comparaison avec les autres langues TupíGuaraní, même avec celles qui lui sont les plus proches.

\section{Références}

Cabral, Ana Suelly. A. C. 2001. O desenvolvimento da marca de objeto de segunda pessoa plural em Tupí-Guaraní. In: Cabral, Ana Suelly A. C., Aryon D. Rodrigues (orgs.), Estudos sobre linguas indígenas I. Atas do I Encontro Internacional do Grupo de Trabalho sobre Linguas Indigenas da ANPOLL. Belém: EDUFPA:167-176.

Cabral, Ana Suelly A. C., e Eliete de Jesus Bararuá Solano. 2006. Mais fundamentos de proximidade genética do Araweté com línguas do sub-ramo V da família Tupí-Guaraní. Estudos da língua(gem), 4. 2:41-65. Vitória da Conquista.

Dixon, R. M. W. 1994. Ergativity. Cambridge: Cambridge University Press.

Jensen, Cheryl. 1998. The use of coreferential and reflexive markers in Tupi-Guarani languages. The Journal of Amazonian languages, 1. 2:1-49. Pittsburgh.

Julião, Maria Risolêta Silva. 2005. Aspects morphosyntaxiques de l'anambe. Toulouse: Université de Toulouse-Le Mirail. Thèse de doctorat.

Leite, Yonne. 2001. O estatuto dos sintagmas nominais de sujeito e de objeto em Tapirapé. In: Queixalós, F. (resp.). Des noms et des verbes en tupiguarani: état de la question. Lincom Europe:87-101. Munique.

Lemaréchal, Alain. 1997. Zero(s). Paris: PUF.

Monserrat, Ruth M. F. e Irmãzinhas de Jesus. 1998. Língua Asuriní do Xingu: observações gramaticais. Altamira: CIMI.

Pereira, Antonia Alves. 2009. Estudo morfossintático do Asuriní do Xingu. Campinas: Universidade Estadual de Campinas. Tese de doutorado. 
Rodrigues, Aryon D. 1981. Estrutura do Tupinambá. Ms.

Rodrigues, Aryon D., Ana Suelly A. C. Cabral (orgs.) 2002. Revendo a classificação interna da família Tupí-Guaraní. In: Linguas Indígenas Brasileiras: Fonologia, gramática e história. Atas do I Encontro Internacional do Grupo de Trabalho sobre Linguas Indígenas da ANPOLL, volume 1. Belém: EDUFPA:327-337.

Seky, Luci. 2000. Gramática do Kamaiurá: língua Tupi-Guarani do Alto Xingu. Campinas, SP: Editora da UNICAMP; São Paulo: Imprensa Oficial.

Vieira, Marcia Dâmaso. 1993. O problema da configurcionalidade na língua Asuriní: uma consequência da projeção dos argumentos no predicado verbal. Campinas: Universidade Estadual de Campinas. Dissertação de mestrado. 